\title{
Cooperative Metal-Ligand Catalyzed Intramolecular Hydroamina- tion and Hydroalkoxylation of Allenes using a Stable Iron Catalyst
}

\author{
Osama El-Sepelgy, ${ }^{*}$ Aleksandra Brzozowska, ${ }^{\ddagger \dagger}$ Jan Sklyaruk, ${ }^{\ddagger \dagger}$ Yoon Kyung Jang, ${ }^{\ddagger}$ Viktoriia Zubar, \\ and Magnus Rueping*,\$, \\ ${ }^{+}$Institute of Organic Chemistry, RWTH Aachen University, Landoltweg 1, 52074 Aachen, Germany, \\ ${ }^{\S}$ King Abdullah University of Science and Technology (KAUST), KAUST Catalysis Center (KCC), Thuwal 23955-6900, \\ Saudi Arabia
}

Supporting Information Placeholder

\begin{abstract}
A new iron-catalyzed chemoselective intramolecular hydroamination and hydroalkoxylation of the readily available $\alpha$-allenic amines and alcohols to valuable unsaturated 5-membered heterocycles, 2,3-dihydropyrrole and 2,3-dihydrofuran is reported. Effective selectivity control is achieved by a metal-ligand cooperative activation of the substrates. The mild reaction conditions and the use of low amounts of an air and moisture stable iron catalyst allow for the hydrofunctionalization of a wide range of allenes bearing different functional groups in good yields in the absence of base or any sensitive additives.
\end{abstract}

Nitrogen and oxygen containing heterocycles are present in most of the naturally occurring and biologically relevant compounds. ${ }^{1}$ Therefore, the development of efficient atomeconomical methods for the synthesis of heterocyclic compounds is of importance. One elegant approach for the synthesis of heterocycles is the transition metal-catalyzed intramolecular hydrofunctionalization of allenes with heteroatom nucleophiles. ${ }^{2}$ Recently, we reported that air and moisture stable iron cyclopentadienone complexes can be applied for carboetherifications and the synthesis of deoxygenated 6membered oxygen-based heterocycles. ${ }^{3}$ This unusual transformation was observed during our investigation of iron cyclopentadienone complexes as hydrogen autotransfer catalysts in dynamic kinetic resolutions. ${ }^{4,5}$

Regarding the intramolecular hydroalkoxylation of $\alpha$ allenic alcohols to 2,5-dihydrofuran a procedure using 5-10 mol $\%$ of $\mathrm{AuCl}_{3}$ was reported by Krause et.al. ${ }^{6}$ More recently Bäckvall and coworkers applied $4 \mathrm{~mol} \%$ of a ruthenium based catalyst to achieve the intramolecular addition reaction. ${ }^{7}$ In addition to these preliminary studies the intramolecular hydrofunctionalization of allenes with $N$-nucleophiles has been explored. This transformation leads to valuable dihydropyrroles, a ring system which is ubiquitous in natural products and is frequently used as a precursor in the synthesis of natural products and bioactive molecules, such as serotonin reuptake inhibitor from Eli Lilly. ${ }^{8}$ So far the endo-cyclization of allene amines was achieved using silver ${ }^{9}$ and gold(III) ${ }^{10}$ catalysts. In analogy to the hydroalkoxylations, these reactions afford the 2,5-dihydro-pyrrole regioisomer.

To the best of our knowledge, a catalytic intramolecular hydroamination of allenic amines to cyclic enamines has not been reported, although the products would be valuable intermediates which could be easily modified.

Therefore, we decided to examine whether the readily available and stable iron cyclopentadienone catalysts could be applied as general catalysts for the intramolecular hydrofunctionalization of allenes.

Scheme 1: Iron-catalyzed hydroamination and hydroalkoxylation of allenes.

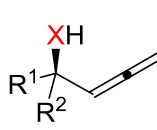

$X=O, N P G$
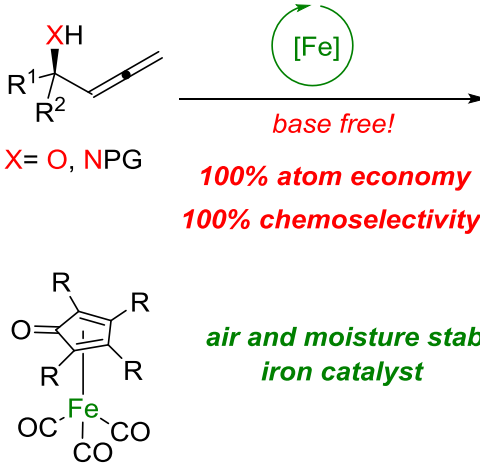

Fe catalyst iron catalyst

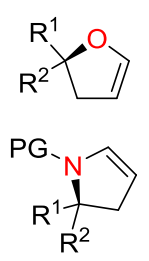

(30 examples) air and moisture stable

Herein, we report a general and efficient endohydrofunctionalization of allenes with nitrogen and oxygen nucleophiles to afford useful 2,3-dihydropyrroles and 2,3dihydrofurans (Scheme 1).

Our studies began with the examination of different cyclopentadienone iron catalysts for the intramolecular hydroamination of allenes. From the outset we focused on 
readily removable amine protecting groups, such as the Cbzprotected amine 1a.

The best results were achieved with the tetraphenyl substituted cyclopentadienone iron tricarbonly catalyst [Fe]. This air and moisture stable complex can be easily synthetized by reaction of two commercially available inexpensive substrates, tetraphenylcyclopentadienone and iron penta-carbonyl. ${ }^{11}$ [Fe] can be activated in situ by oxidative decarbonylation using trimethylamine $\mathrm{N}$-oxide. The hydroamination of the $\mathrm{Cbz}-$ allenic amine 1a was subsequently examined using $5 \mathrm{~mol} \%$ of [Fe] at $70{ }^{\circ} \mathrm{C}$ in different solvents (Table 1). When the reaction was performed in toluene the desired enamine $\mathbf{2 a}$ was obtained in $72 \%$ yield (Table 1, entry 1). The application of hexane as a solvent lead to only $40 \%$ yield (Table 1 , entry 2 ).

Table 1. Optimization of the reaction conditions ${ }^{\mathrm{a}}$

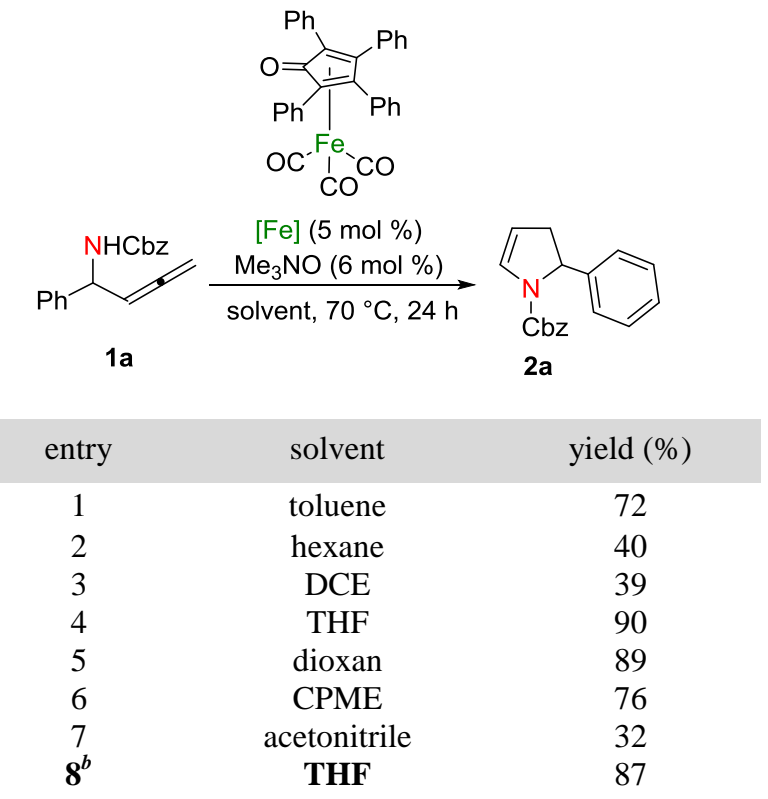

${ }^{a}$ Reaction conditions: $1(0.1 \mathrm{mmol}),[\mathbf{F e}](5 \mathrm{~mol} \%)$ and $\mathrm{Me}_{3} \mathrm{NO}(6 \mathrm{~mol} \%)$ in solvent $(0.5 \mathrm{~mL})$ were stirred at $70{ }^{\circ} \mathrm{C}$ for $24 \mathrm{~h}$ in a Schlenk tube under an inert atmosphere. Yields determined by ${ }^{1} \mathrm{H}$ NMR using mesitylene as internal standard. ${ }^{b} \mathbf{1}(0.5$ mmol) in $1 \mathrm{~mL}$ of THF. Yield of product after chromatography. $\mathrm{CPME}=$ cyclopropyl methyl ether

Furthermore, the halogenated solvent, dichloroethane gave the same product in low yield (Table 1, entry 3). Further screening of solvents showed that ethers are superior to nonpolar solvents (Table 1, entries 4-6). The best results were obtained when the reaction was performed in tetrahydrofuran and the desired product was isolated in $87 \%$ yield (Table 1, entries 4,8 ). Use of acetonitrile gave only $32 \%$ yield which can be explained by the strong coordination of the acetonitrile with the active iron specie (Table 1, entry 7).

In order to demonstrate the broad practicability of our newly developed method, we applied the optimized cyclization protocol to various allenic amines (Table 2) bearing different functionalities. Thus, various 2,3-dihydropyrroles can be effectively obtained in good yields. Next to 2,3-dihydropyrrole 2a, which was isolated in $87 \%$ yield (Table 2, entry 1), dihydropyrroles $\mathbf{b}-\mathbf{f}$ bearing electron withdrawing substituents in para position of the arene were also obtained in very good yields (Table 2, entries 2-5). Allenic amine 1f with an electron withdrawing substituent in ortho position gave the corresponding pyrrole in $78 \%$ yield (Table 2, entry 6 ), while 19 with the electron donating methyl group was cyclized in good yield of $71 \%$ (Table 2, entry 7). Furthermore, the 2-naphtyl substituted 2,3-dihydropyrrol $\mathbf{2} \mathbf{g}$ was isolated in $85 \%$ yield (Table 2, entry $8)$. Our method could also be applied to the aliphatic allenic amines 1i-11, leading to the corresponding heterocycles $\mathbf{2 i - 1}$ in moderate to good yields (Table 2, entries 9-12). This cyclic enamine $2 \mathbf{l}$ is of relevance as it can be used for the synthesis of the pyrrole analogues of several antibiotics. ${ }^{12}$

Table 2. Iron catalyzed hydroamination of $\alpha$-allenic amines. ${ }^{a}$
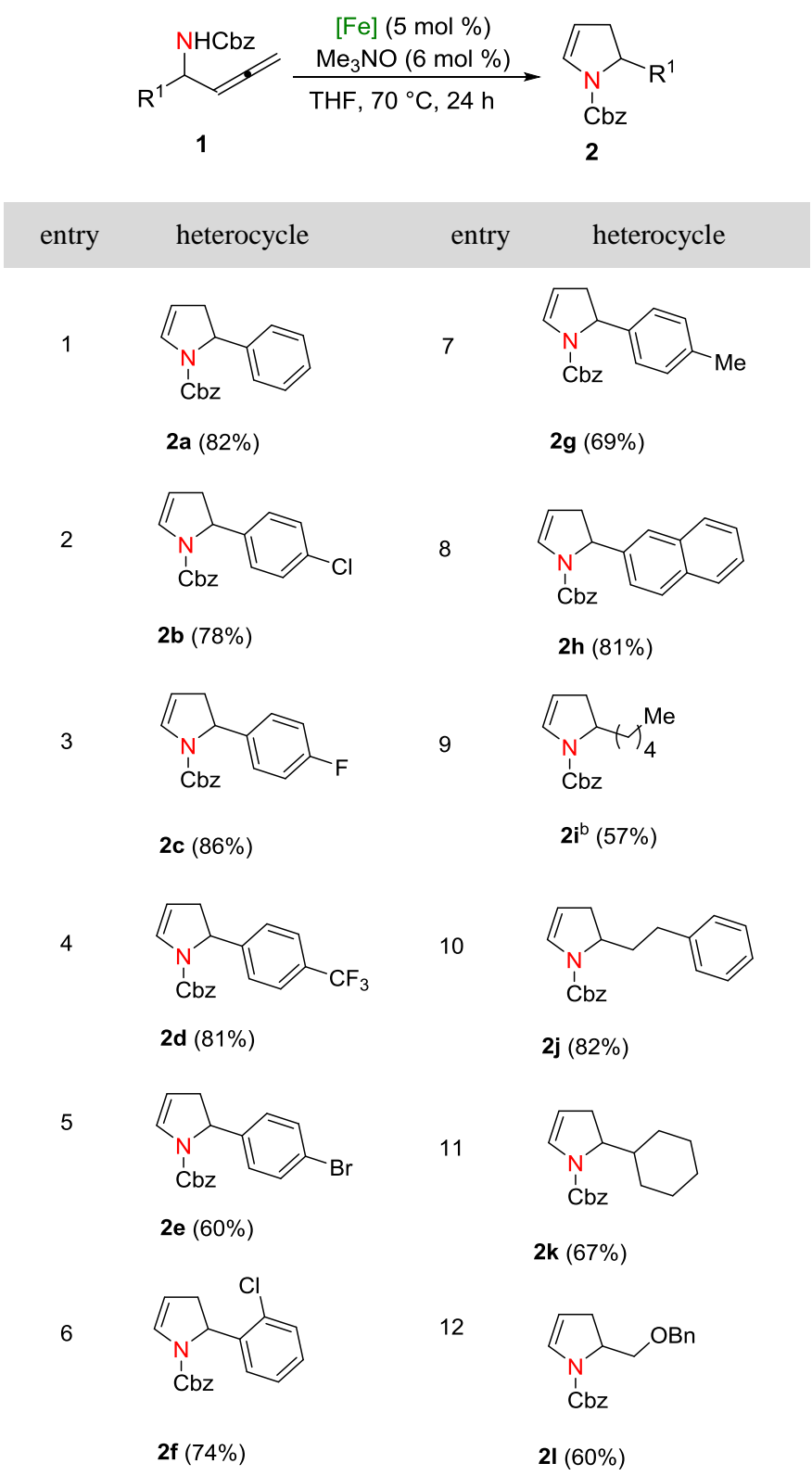

${ }^{a}$ Reaction conditions: $1(0.5 \mathrm{mmol}),[\mathrm{Fe}](5 \mathrm{~mol} \%)$ and $\mathrm{Me}_{3} \mathrm{NO}$ $(6 \mathrm{~mol} \%)$ in THF $(1 \mathrm{~mL})$ were stirred at $70{ }^{\circ} \mathrm{C}$ for $24 \mathrm{~h}$ in a Schlenk tube under inert atmosphere. Isolated yields are given. ${ }^{b}[\mathbf{F e}](10 \mathrm{~mol} \%)$ and $\mathrm{Me}_{3} \mathrm{NO}(12 \mathrm{~mol} \%)$.

Following the successful development of the intramolecular hydroamination of allenes to provide 2,3-dihydropyrroles, we 
became interested in the generalization of this new protocol by applying $\alpha$-allenic alcohols.

Table 3. Iron catalyzed hydroalkoxylation of allenic alcohols ${ }^{a}$.

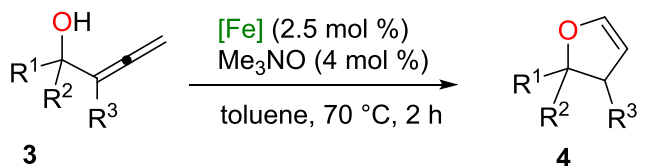

(9)

${ }^{a}$ Reaction conditions: $3(1 \mathrm{mmol}),[\mathbf{F e}](2.5 \mathrm{~mol} \%)$ and $\mathrm{Me}_{3} \mathrm{NO}$ $(4 \mathrm{~mol} \%)$ in toluene $(1 \mathrm{~mL})$ were stirred at $70{ }^{\circ} \mathrm{C}$ for $2 \mathrm{~h}$ in a
Schlenk tube under an inert atmosphere. Isolated yields are shown.

This hydroalkoxylation would result in valuable 2,3dihydrofurans. To our delight, $\alpha$-allenic alcohols could also successfully be applied. Indeed, if compared to $\alpha$-allenic amines the reaction proceeded faster and, thus, a reduction to $2.5 \mathrm{~mol} \%$ of the $[\mathbf{F e}]$ catalyst was feasible to provide the product in $2 \mathrm{~h}$. It is noteworthy that the required catalyst loading for the previously reported Ru-protocol was 4 mol \%, which further highlights the advantage of the current basemetal alternative. We then investigated the scope of ironcatalyzed intramolecular hydroalkoxylation of allenic alcohols (Table 3). Various aryl-substituted allenic alcohols could be applied, showing the generality of our method. All investigated substrates bearing electron-withdrawing groups and electron-donating groups $\mathbf{3 a - 3 g}$ reacted smoothly providing the corresponding 2,3-dihydrofuranes in high yields (Table 3, entries 1-7). Furthermore, the carboetherification of the naphthyl substituted allenic alcohol $\mathbf{3 h}$ proceeds well to afford the corresponding furan in $95 \%$ yield (Table 3 , entry 8 ). Moreover, several cyclic and acyclic aliphatic derivatives 3i-3l were cyclized to the unsaturated furans $4 \mathbf{i}-4 \mathbf{l}$ in good yields (Table 3, entries 9-12). Importantly, deoxysugar $\mathbf{4 1}$ is of high relevance as it is industrially used in the synthesis of clinically relevant antibiotics such as AZT, d4T and Puromycin. ${ }^{12}$ Interestingly, the unsaturated furan bearing conjugated double bond $\mathbf{4 m}$ was also obtained in good yield (Table 3, entry 13). The new protocol was also found to be applicable to heteroarenes as demonstrated for the derivatives $\mathbf{4 n}$ and $\mathbf{4 o}$ (Table 3, entries 14 and 15). Importantly, our catalytic system has been useful for the construction of the spirocyclic ethers such as $\mathbf{4 p}$ (Table 3 , entry 16). Moreover, the hydroalkoxylation of the tertiary alcohol $\mathbf{3 q}$ gave the furan $\mathbf{4 q}$ bearing a quaternary carbon atom (Table 3, entry 17). Following the successful demonstration of the applicability of the newly developed iron catalyzed hydroalkoxylation of 1-substituted allenols, we turned our attention to the use of 1,2-disubstituted $\alpha$-allenols which should provide two different diastereomers.

Scheme 2: Proposed reaction mechanism.

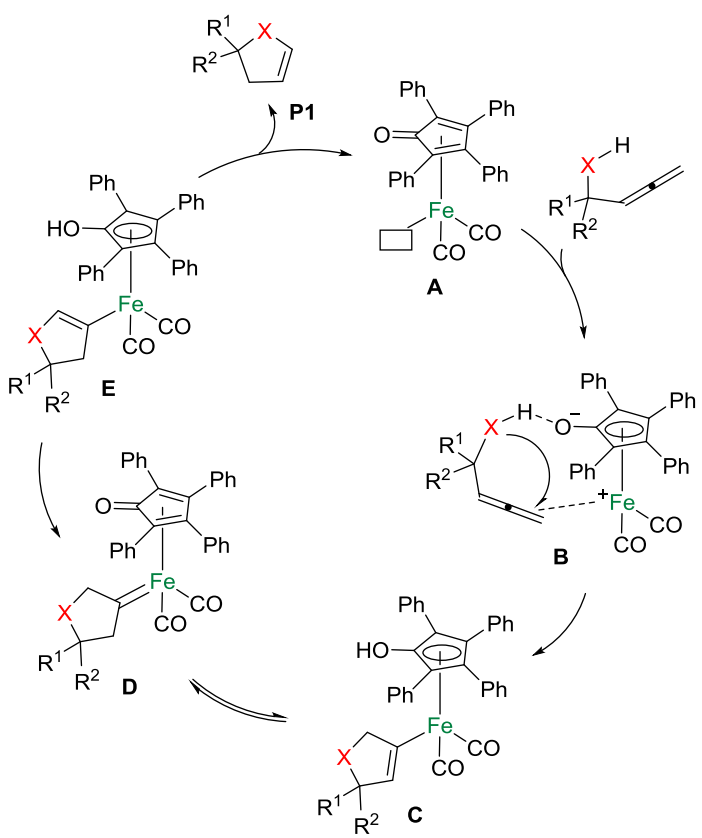


Applying the same protocol, we could convert the disubstituted allenic alcohol $3 \mathbf{r}$ to the corresponding disubstituted dihydrofurane in $68 \%$ yield and $4: 1$ diastereomeric ratio demonstrating a new and efficient method for the construction of cyclic ethers with two chiral centers (Table 3, entry 18).

Based on the results and our previous work, ${ }^{3}$ we propose that the reaction is catalyzed by the in situ generated $16 e$ iron species $\mathbf{A}$ which is obtained by decarbonylation of the iron tricarbonyl pre-catalyst [Fe]. The bifunctional catalytic species A bearing a non-innocent ligand ${ }^{13}$ exhibits a dual catalytic task. While the $\mathrm{C}=\mathrm{O}$ group of the ligand activates the nucleophile through hydrogen bonding the iron metal center activates the terminal double bond of allene by coordination. The activated $\mathrm{O}$-or $\mathrm{N}$-nucleophile of $\mathbf{B}$ can undergo intramolecular attack with transfer of a hydrogen atom to the cyclopentadienone ligand, producing the iron vinylidene intermediate $\mathbf{C}$. The next steps involves two consecutive hydrogen transfers, leading to the isomerization of the iron vinylidene intermediate $\mathbf{C}$ into the more stable iron vinylidene intermediate $\mathbf{E}$ via the iron carbene intermediate D. Finally, the desired product is selectively formed via protodemetallation of the intermediate $\mathbf{E}$ (Scheme 2).

In conclusion, we report the first example of an intramolecular hydroamination of allenic amines to enamines as well as an efficient base metal catalyzed hydroalkoxylation of $\alpha$-allenic alcohols to valuable cyclic enol ethers. ${ }^{7 \mathrm{~b}}$ The general applicability of this protocol is highlighted by the synthesis of 30 unsaturated heterocycles with good yields and excellent chemoselectivity. The key to achieve this unusual selectivity is the use of an iron based metal-ligand catalyst which can activate the substrate in a dual catalytic fashion. The absence of any sensitive chemicals as well as base additives is an additional advantage of the reported protocol. We believe that this strategy will find practical application in the synthesis of bioactive molecules and natural products.

\section{ASSOCIATED CONTENT}

\section{Supporting Information}

The Supporting Information is available free of charge on the ACS Publications website at DOI: 10.1021/acs.orglett.xxxxx

\section{AUTHOR INFORMATION}

\section{Corresponding Author}

*Email: osama.elsepelgy@ rwth-aachen.de

*Email: magnus.rueping@ rwth-aachen.de

\section{ORCID}

Osama El-Sepelgy: 0000-0003-3131-4988

Magnus Rueping: 0000-0003-4580-5227

\section{Author Contributions}

${ }^{\dagger}$ A.B. and J.S. contributed equally to this work

\section{Note}

The authors declare no competing financial interest

\section{ACKNOWLEDGMENT}

J.S thanks FCI for the pre-doctoral fellowship.

\section{REFERENCES}

(1) Eicher, T.; Hauptmann, S.; Speicher, A.; The Chemistry of Heterocycles; Wiley-VCH, Weinheim, 2003.

(2) (a) Reissig, H.-U.; Zimmer, R.; Allenes in Multicomponent Synthesis of Heterocycles. In Multicomponent Reactions in Organic Synthesis; Zhu, J.; Wang, Q.; Wang, M.-X. Eds.; Wiley-VCH Verlag GmbH \& Co. KGaA, Weinheim, Germany, 2014. (b) Modern Allene Chemistry; Krause, N.; Hashmi, A. S. K., Eds.; Wiley-VCH Verlag GmbH \& Co. KGaA, Weinheim, Germany, 2004, Vol 1 and 2; for reviews, see: (c) Ma, S. Acc. Chem Res. 2003, 36, 701-712. d) Widenhoefer, R. A. Chem. Eur. J. 2008, 14, 5382-5391. (e) Ma, S. Acc. Chem Res. 2009, 42, 1679-1688. (f) Krause, N.; Winter, C. Chem. Rev. 2011, 111, 1994-2009.

(3) El-Sepelgy, O.; Brzozowska, A.; Azorfa, L. M.; Jang, Y. K.; Cavallo, L.; Rueping, M. Angew. Chem. Int. Ed. 2017, 56, 1486314867.

(4) (a) El-Sepelgy, O.; Alandini, N.; Rueping, M. Angew. Chem. Int. Ed. 2016, 55, 13602-13605. (b) El-Sepelgy, O.; Brzozowska, A.; Rueping, M. ChemSusChem 2017, 10, 1664-1668. (c) Gustafson, K. P. J.; Guđmundsson, A.; Lewis, K.; Bäckvall, J.-E. Chem. Eur. J. 2017, 23, 1048-1051.

(5) (a) Iron Catalysis in Organic Chemistry: Reactions and applications; Plietker, B., Ed.; ed.2, Wiley-VCH Verlag GmbH \& Co. KGaA, Weinheim, Germany, 2008; for recent reviews on iron catalysis, see: (b) Bauer, I.; Knölker, H.-J. Chem. Rev. 2015, 115, 31703387. for seminal work on the use of the iron carbonyl complexes for hydrogenation, see: (c) Casey, C. P.; Guan, H. J. Am. Chem. Soc. 2007, 129, 5816-5817. (d) Casey, C. P.; Guan, H. J. Am. Chem. Soc. 2009, 131, 2499-2507. for hydrogen borrowing, see: (e) Yan, T.; Feringa, B. L.; Barta, K. Nat. Commun. 2014, 5, 5602. (f) Rawlings, A. J.; Diorazio, L. J.; Wills, M. Org. Lett. 2015, 17, 1086-1089. for a recent review on iron cyclopentadienone, see: (g) Quintard, A.; Rodriguez, J. Angew. Chem. Int. Ed. 2014, 53, 4044-4055. For a review on iron catalyzed hydrofunctionalization see: (h) Greenhalgh, M. D.; Jones, A. S.; Thomas, S. P., ChemCatChem 2015, 7, 190-222.

(6) (a) Hoffmann-Röder, A.; Krause, N.; Org. Lett. 2001, 16, $2537-$ 2538. (b) Eom, D.; Kang, D.; Lee, P. H. J. Org. Chem. 2010, 75, 7447-7450

(7) (a) Yang, B.; Zhu, C.; Qiu, Y.; Bäckvall, J.-E. Angew.Chem. Int.Ed. 2016, 55, $5568-5572$. During the final preparation of this of this manuscript, the same group reported the use of $5 \mathrm{~mol} \%$ of a related iron catalyst and $100 \mathrm{~mol} \%$ of base for the cycloisomerization of $\alpha$-allenic alcohols to 2,3-dihydrofuran: (b) Guđmundsson, A.; Gustafson, K. P. J.; Mai, B. K.; Yang, B.; Himo, F.; Bäckvall, J.-E. ACS Catal. 2018, 8, 12-16.

(8) (a) L. W. Hertel and Y. C. Xu, Eli Lilly and Company (US), Patent: US6353008 B1, 2002, 14 (b) Wegner, J.; Ley, S. V.; Kirschning, A.; Hansen, A.-L.; Garcia, J. M.; Baxendale, I. R. Org. Lett. 2012, 3 , 696-699. (c) Myers, A. G.; Herzon, S. B. J. Am. Chem. Soc. 2005, 127, 5342-5344. (d) Zhou, J.; Wu, C. J. Am. Chem. Soc. 2014, 136, 650-652. (e) Xu, H.; Zuend, S. J.; Woll, M. G.; Tao, Y.; Jacobsen, E. N. Science, 2010, 327. (f) Zhang, H.; Jeon, K. O.; Hay, E. B.; Geib, S. J; Curran, D. P.; LaPorte, M. G. Org. Lett. 2014, 16, 94-97.

(9) (a) Dieter, R. K.; Yu, H. Org. Lett. 2001, 24, 3855-3858. (b) Billet, M.; Schoenfelder, A.; Klotz, P.; Mann, A. Tetrahedron Lett. 2002, 43, 1453-1456.

(10) (a) Morita, N.; Krause, N. Org. Lett. 2004, 22, 4121-4123; for further applications, see: (b) Lee, P. H.; Kim, H.; Lee, K.; Kim, M.; Noh, K.; Kim, H.; Seomoon, D. Angew. Chem. Int. Ed. 2005, 44, 1840-1843; (c) Alcaide, B.; Almendros, P.; Martinez del Campo, T.; Redondo, M. C.; Fernandez, I. Chem. Eur. J. 2011, 17, 15005-15013.

(11) (a) Weiss, E.; Hübel, W. J. Inorg. Nucl. Chem. 1959, 11, 42; (b) Weiss, E.; Merényi, R.; Hübel, W. Chem. Ber. 1962, 95, 1170

(12) McDonald, F. E.; Gleason, M. M. J. Am. Chem. Soc. 1996, 118,6648 .

(13) (a) Khusnutdinova, R.; Milstein, D. Angew. Chem. Int. Ed. 2015, 54, 12236-12273; (b) Luca, O. R.; Crabtree, R. H. Chem. Soc. Rev. 2013, 42, 1440-1459; (c) Lyaskovskyy, V.; de Bruin, B. ACS Catal. 2012, 2, 270-279. 


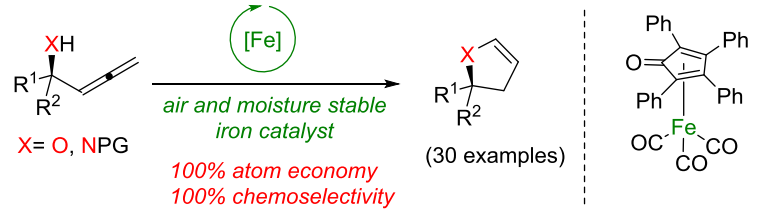

